\title{
TRACE FORMS OF SYMBOL ALGEBRAS
}

\author{
RONAN FLATLEY
}

\begin{abstract}
Aвstract. Let $S$ be a symbol algebra. The trace form of $S$ is computed and it is shown how this form can be used to determine whether $S$ is a division algebra or not. In addition, the exterior powers of the trace form of $S$ are computed.
\end{abstract}

\section{INTRODUCTION}

Let $n$ be an arbitrary positive integer and let $K$ be a field containing a primitive $n$-th root of unity $\omega$. Unless stated otherwise, we assume throughout this paper that $\operatorname{char}(K)$ is different from 2 and does not divide $n$. Let $K^{\times}=K \backslash\{0\}$. Let $a, b \in K^{\times}$and let $S$ be the algebra over $K$ generated by elements $x$ and $y$ where

$$
x^{n}=a, \quad y^{n}=b \quad \text { and } \quad y x=\omega x y .
$$

We call this algebra a symbol algebra (see [4, Chapter 1, §2]). Note that in [2, §11], Draxl calls such an algebra a power norm residue algebra, denoting it as $(a, b ; n, K, \omega)$ and shows it to be a central simple algebra over $K$ of degree $n$. Quaternion algebras are the symbol algebras of degree 2 .

Let $A$ be a central simple algebra of degree $n$ over a field $K$ of characteristic different from 2 . We write $T_{A}: A \rightarrow K$ for the quadratic trace form

$$
T_{A}(z)=\operatorname{Trd}_{A}\left(z^{2}\right) \quad \text { for } z \in A,
$$

where $\operatorname{Trd}_{A}$ is the reduced trace of $A$. The main purpose of this paper is to compute the trace form of a symbol algebra $S$ and to show how the form determines if the algebra is division.

Notation and terminology is borrowed from Lam's book [5] and Scharlau's book [11]. A diagonalised quadratic form over $K$ with coefficients $a_{1}, \ldots, a_{m} \in K^{\times}$is denoted by $\left\langle a_{1}, \ldots, a_{m}\right\rangle$. The hyperbolic plane $\langle 1,-1\rangle$ is denoted by $\mathbb{H}$. If $\varphi$ and $\psi$ are forms over $K$ then $\varphi \simeq \psi$ means that these forms are isometric. The Witt index of $\varphi$ is denoted by $i_{W}(\varphi)$ and the anisotropic part of $\varphi$ by $\varphi_{a n}$, so that we have $\varphi \simeq \varphi_{a n} \perp(i \times \mathbb{H})$ where $i=i_{W}(\varphi)$. The tensor product of the 1-dimensional form $\langle n\rangle$ with $\varphi$ is denoted $\langle n\rangle \varphi$ to be distinguished from $n$ copies of $\varphi$ which is written as $n \times \varphi$.

Date: November 27, 2008.

2000 Mathematics Subject Classification. Primary: 11E81; Secondary: 16K20.

Key words and phrases. symbol algebra, trace form, exterior power. 


\section{Symbol algEbras AND THEIR TRACE FORMS}

Let $S$ be the symbol algebra $(a, b ; K, n, \omega)$ with basis $\left\{x^{i} y^{j}\right\}, 0 \leq i, j \leq n-1$.

Proposition 2.1. We have

$$
\begin{array}{ll}
\text { (i) } T_{S} \simeq\langle n\rangle \perp \frac{n^{2}-1}{2} \times \mathbb{H} & \text { for } n \text { odd. } \\
\text { (ii) } T_{S} \simeq\langle n\rangle\left\langle 1, a, b,(-1)^{n / 2} a b\right\rangle \perp \frac{n^{2}-4}{2} \times \mathbb{H} & \text { for } n \text { even. }
\end{array}
$$

Proof. Let $\phi_{T_{S}}$ be the symmetric bilinear form associated with $T_{S}$. Consider $\left\{x^{i} y^{j}\right\}$, $0 \leq i, j \leq n-1$, the set of $n^{2}$ basis elements of $S$. Consider the left regular matrix representation of each such element under the isomorphism used in the definition of the trace map. Easy, but tedious arguments, and switching from the trace to the reduced trace show that

$$
\phi_{T_{S}}\left(x^{i} y^{j}, x^{i} y^{j}\right)=\operatorname{Trd}_{S}\left(\left(x^{i} y^{j}\right)^{2}\right)=0
$$

unless we have of the following cases:

$$
\begin{array}{ll}
i=j=0, & \text { in which case the reduced trace is } n ; \\
i=0 \text { and } j=\frac{n}{2}, & \text { in which case the reduced trace is } n b ; \\
i=\frac{n}{2} \text { and } j=0, & \text { in which case the reduced trace is } n a ; \\
i=\frac{n}{2} \text { and } j=\frac{n}{2}, & \text { in which case the reduced trace is }(-1)^{\frac{n}{2}} n a b .
\end{array}
$$

Clearly, the latter three cases only arise for $n$ even.

(i) Le $i=j=0$. Then $\phi_{T_{S}}(1,1)=n$, as mentioned above. Now let $1 \leq i, j \leq n-1$. There are $\frac{n^{2}-1}{2}$ pairs $\left(x^{i} y^{j}, x^{n-i} y^{n-j}\right)$ and we have

$$
\phi_{T_{S}}\left(x^{i} y^{j}, x^{n-i} y^{n-j}\right)=\operatorname{Trd}_{S}\left(x^{i} y^{j} x^{n-i} y^{n-j}\right)=\operatorname{Trd}_{S}\left(\omega^{j(n-i)} x^{n} y^{n}\right)=n \omega^{-i j} a b .
$$

Each pair contributes a $2 \times 2$ block in the Gram matrix of $\phi_{T_{S}}$, as follows:

$$
\begin{array}{c|cc}
\phi_{T_{S}}(-,-) & x^{i} y^{j} & x^{n-i} y^{n-j} \\
\hline x^{i} y^{j} & 0 & n \omega^{-i j} a b \\
x^{n-i} y^{n-j} & n \omega^{-i j} a b & 0
\end{array}
$$

Each such block corresponds to a 2-dimensional hyperbolic plane which is a direct summand of $T_{S}$. The Gram matrix will have exactly one non-zero entry in each row. Hence $T_{S} \simeq\langle n\rangle \perp \frac{n^{2}-1}{2} \times \mathbb{H}$.

(ii) The subset $\left\{1, x^{n / 2}, y^{n / 2}, x^{n / 2} y^{n / 2}\right\}$ of basis elements of $S$ gives rise to the quadratic form $\langle n\rangle\left\langle 1, a, b,(-1)^{n / 2} a b\right\rangle$. By placing the other $n^{2}-4$ basis elements into ordered pairs of the form $\left(x^{i} y^{j}, x^{n-i} y^{n-j}\right)$, we get that $\phi_{T_{S}}$ maps each pair to $n \omega^{-i j} a b$ as seen 
in the proof of $(i)$. So we get $\frac{n^{2}-4}{2}$ hyperbolic planes as direct summands. Hence $T_{S} \simeq\langle n\rangle\left\langle 1, a, b,(-1)^{n / 2} a b\right\rangle \perp \frac{n^{2}-4}{2} \times \mathbb{H}$.

By way of example, the matrix of $T_{S}$ when $n=3$ is as follows:

$$
\left[\begin{array}{cccccccc}
3 & & & & & & \\
& 0 & 3 a & & & & & \\
3 a & 0 & 0 & 3 b & & & \\
& & 3 b & 0 & & & \\
& & & & 0 & 3 \omega^{2} a b & & \\
& & & 3 \omega^{2} a b & 0 & 0 & 3 \omega a b
\end{array}\right] .
$$

The matrix is computed for the basis elements $\left\{1, x, x^{2}, y, y^{2}, x y, x^{2} y^{2}, x^{2} y, x y^{2}\right\}$ and each blank entry in the matrix is zero.

\section{Further RESUlts WhEN $\operatorname{deg}_{K} S$ IS ODD}

We now return to the case when $n$ is odd and show that we can improve upon the formula deduced for $T_{S}$. We require the following two propositions.

Proposition 3.1. Let $n$ be odd. Then $\langle n\rangle \simeq\left\langle(-1)^{\frac{n-1}{2}}\right\rangle$.

Proof. We recall the following definitions from classical number theory: for $p$ an odd prime

$$
\tau_{p}:=\sum_{i=1}^{p-1}\left(\frac{i}{p}\right) \omega^{i}
$$

where $\left(\frac{i}{p}\right)$ is the Legendre symbol and

$$
p^{*}:=\left(\frac{-1}{p}\right) p
$$

We have the theorems (see [8], for example)

$$
p^{*}=\tau_{p}^{2} \text { and }\left(\frac{-1}{p}\right)=(-1)^{\frac{p-1}{2}}
$$

and the facts

$$
p_{1} \equiv 1 \quad(\bmod 4) \Leftrightarrow p_{1}^{*}=p_{1} \quad \text { and } \quad p_{2} \equiv 3 \quad(\bmod 4) \Leftrightarrow p_{2}^{*}=-p_{2} .
$$

Suppose $n$ is prime. Then $\tau_{n}^{2}=n^{*}=\left(\frac{-1}{n}\right) n=(-1)^{\frac{n-1}{2}} n$. On the other hand suppose $n$ is compound. Write $n=\prod_{j=1}^{t} p_{j}$ where the $p_{j}$ are primes, not all necessarily different and $t>1$. If $n \equiv 1(\bmod 4)$ then

$$
n=\prod_{j=1}^{t} p_{j}^{*}=(-1)^{\frac{n-1}{2}} \prod_{j=1}^{t} p_{j}^{*}=(-1)^{\frac{n-1}{2}}\left(\prod_{j=1}^{t} \tau_{j}\right)^{2} .
$$


Otherwise, if $n \equiv 3(\bmod 4)$ then

$$
n=-\prod_{j=1}^{t} p_{j}^{*}=(-1)^{\frac{n-1}{2}} \prod_{j=1}^{t} p_{j}^{*}=(-1)^{\frac{n-1}{2}}\left(\prod_{j=1}^{t} \tau_{j}\right)^{2} .
$$

Hence $\langle n\rangle \simeq\left\langle(-1)^{\frac{n-1}{2}}\right\rangle$.

Proposition 3.2. Let $n$ be odd. Then $n \times\langle 1\rangle \simeq\left\langle(-1)^{\frac{n-1}{2}}\right\rangle \perp \frac{n-1}{2} \times \mathbb{H}$.

Proof. Recall that the level of a field $F$, denoted $s(F)$, is the least number of squares required to sum to -1 in $F$ or $\infty$ if no such number exists. Since $K$ contains a primitive $n$-th root of unity, $s(K) \in\{1,2,4\}$. If $n \equiv 3$ or $5(\bmod 8)$, then $n$ has a prime divisor $p$ such that $p \equiv 3$ or $5(\bmod 8)$. In this case we have $s(K)=2$ by [3]. Thus, $2 \times\langle 1\rangle \simeq$ $2 \times\langle-1\rangle$ which implies

$$
\begin{array}{rlrl}
n \times\langle 1\rangle & \simeq 3 \times\langle 1\rangle \perp \frac{n-1}{2} \times \mathbb{H} & & \text { if } n \equiv 3 \quad(\bmod 8) \\
& \simeq\langle-1\rangle \perp \frac{n-1}{2} \times \mathbb{H} & & \\
n \times\langle 1\rangle & \simeq 5 \times\langle 1\rangle \perp \frac{n-7}{2} \times \mathbb{H} & & \text { if } n \equiv 5 \quad(\bmod 8) \\
& \simeq\langle 1\rangle \perp \frac{n-1}{2} \times \mathbb{H} . &
\end{array}
$$

In the remaining cases of interest we have $s(K) \leq 4$, i.e. $4 \times\langle 1\rangle \simeq 4 \times\langle-1\rangle$. Therefore,

$$
\begin{aligned}
n \times\langle 1\rangle & \simeq\langle 1\rangle \perp \frac{n-1}{2} \times \mathbb{H} & & \text { if } n \equiv 1 \quad(\bmod 8) \\
n \times\langle 1\rangle & \simeq 7 \times\langle 1\rangle \perp \frac{n-7}{2} \times \mathbb{H} & & \text { if } n \equiv 7 \quad(\bmod 8) \\
& \simeq\langle-1\rangle \perp \frac{n-1}{2} \times \mathbb{H} . & &
\end{aligned}
$$

Corollary 3.3. For $n$ odd, $T_{S} \simeq n^{2} \times\left\langle(-1)^{\frac{n-1}{2}}\right\rangle$.

Proof. Propositions 2.1 $i$ ) and 3.1 show that for $n$ odd,

$$
\begin{aligned}
T_{S} & \simeq\langle n\rangle \perp \frac{n^{2}-1}{2} \times \mathbb{H} \\
& \simeq\left\langle(-1)^{\frac{n-1}{2}}\right\rangle \perp \frac{n^{2}-1}{2} \times \mathbb{H} .
\end{aligned}
$$

Then by Proposition 3.2 and the fact that

$$
n^{2} \times\left\langle(-1)^{\frac{n-1}{2}}\right\rangle=n \times\left\langle(-1)^{\frac{n-1}{2}}\right\rangle \otimes n \times\langle 1\rangle,
$$

we get $T_{S} \simeq n^{2} \times\left\langle(-1)^{\frac{n-1}{2}}\right\rangle$. 
Remark 3.4. For $n$ odd, we could also write $T_{S} \simeq n \times\langle 1\rangle \perp \frac{n^{2}-n}{2} \times \mathbb{H}$ which can be deduced from the calculation in the split case, see [6], together with Springer's Theorem on odd degree extensions (see [5, p.194], for example).

4. TRACE FORM CRITERIA TO DETERMINE IF A SYMBOL ALGEBRA IS DIVISION

Proposition 4.1. If $n \equiv 2(\bmod 4)$ then the quaternion algebra $(a, b)_{K}$ is contained in $S$.

Proof. Consider the elements $x^{n / 2}, y^{n / 2} \in S$. Then $\left(x^{n / 2}\right)^{2}=a,\left(y^{n / 2}\right)^{2}=b$ and $y^{n / 2} x^{n / 2}=\left(\omega^{n / 2}\right)^{n / 2} x^{n / 2} y^{n / 2}=-x^{n / 2} y^{n / 2}$.

Proposition 4.2. If $n \equiv 2(\bmod 4)$ and $T_{S}$ is hyperbolic then -1 is a square in $K$ and $S$ is not a division algebra.

Proof. Suppose $T_{S}$ is hyperbolic. By Proposition 4.1, $Q:=(a, b)_{K} \subset S$. Since $n$ is even we have from Proposition 2.1 (ii) that

$$
T_{S} \simeq\langle n\rangle\left\langle 1, a, b,(-1)^{n / 2} a b\right\rangle \perp \frac{n^{2}-4}{2} \times \mathbb{H} \simeq\langle n\rangle\langle 1, a, b,-a b\rangle \perp \frac{n^{2}-4}{2} \times \mathbb{H} .
$$

Thus, by our assumption, $\langle 1, a, b,-a b\rangle \simeq 2 \times \mathbb{H}$ and by evaluating determinants we get $\langle-1\rangle \simeq\langle 1\rangle$. Thus, the norm form of $Q, N_{Q}:=\langle 1,-a,-b, a b\rangle$ is hyperbolic. This implies that $Q$, and thus $S$, contains zero divisors. Therefore, $S$ is not division.

Proposition 4.3. Let $K$ be a field such that $-1 \in K^{\times 2}$. Let $A$ be any central simple algebra over $K$. Let $n:=\operatorname{deg}_{K} A$ be a power of 2 . If $T_{A}$ is not hyperbolic, then $A$ is a division algebra.

Proof. Suppose $A$ is not a division algebra. Then $A \cong M_{r}(D)$ for some integer $r>1$ and some division algebra $D$ over $K$. Now $T_{A} \simeq T_{M_{r}(D)} \simeq T_{M_{r}(K) \otimes_{K} D} \simeq T_{M_{r}(K)} \otimes T_{D} \simeq$ $r \times\langle 1\rangle \otimes T_{D}$ by [6, Lemma 1.2]. Since $n$ is a 2-power, $r$ must be even and since $-1 \in K^{\times 2}$, we have that $T_{A}$ is hyperbolic.

\section{EXTERIOR POWERS OF THE TRACE FORM OF A SYMBOL ALGEBRA}

Bourbaki defined the concept of exterior power of a symmetric bilinear form in [1, IX, §1, (37)]. McGarraghy derived basic properties of such forms in the WittGrothendieck ring of a field in [7]. We present some key definitions and results for exterior powers from McGarraghy's paper as well as some new results. In all cases, $K$ denotes a field of characteristic different from 2.

Definition 5.1. Let $\varphi: V \times V \rightarrow K$ be a bilinear form and let $k$ be a positive integer not greater than $m$. We define the $k$-fold exterior power of $\varphi$,

$$
\Lambda^{k} \varphi: \Lambda^{k} V \times \Lambda^{k} V \rightarrow K
$$

by

$$
\Lambda^{k} \varphi\left(x_{1} \wedge \cdots \wedge x_{k}, y_{1} \wedge \cdots \wedge y_{k}\right)=\operatorname{det}\left(\varphi\left(x_{i}, y_{j}\right)\right)_{1 \leq i, j \leq k}
$$


We define $\Lambda^{0} \varphi:=\langle 1\rangle$, the identity form of dimension 1 . For $k>m$, we define $\Lambda^{k} \varphi$ to be the zero form, since $\Lambda^{k} V=0$ for all $k>m$.

Let $V$ be a vector space of dimension $m$ over $K$. If $k$ is a non-negative integer then the $k$-fold exterior power of $V, \Lambda^{k} V$, has dimension $\left(\begin{array}{l}m \\ k\end{array}\right)$, where we take $\left(\begin{array}{c}m \\ k\end{array}\right)$ to be 0 for all $k>m$. In particular, if $\left\{v_{1}, \ldots, v_{m}\right\}$ is a basis for $V$, then a basis for $\Lambda^{k} V$ is given by the set of $k$-fold wedge products $\left\{v_{i_{1}} \wedge \cdots \wedge v_{i_{k}}: 1 \leq i_{1}<\cdots<i_{k} \leq m\right\}$ and there are $\left(\begin{array}{c}m \\ k\end{array}\right)$ such expressions.

Remark 5.2. We have $\Lambda^{1} \varphi \simeq \varphi$. It is easily seen that $\Lambda^{k} \varphi$ is a bilinear form and is symmetric if $\varphi$ is symmetric. Also, if $q$ is the quadratic form associated to $\varphi$, we write $\Lambda^{k} q$ for the quadratic form associated to $\Lambda^{k} \varphi$.

Proposition 5.3. [7, Proposition 4.1] Let $V$ be a vector space over $K$ with $\operatorname{dim}_{K} V=m$. Let $\varphi$ be a symmetric bilinear form over $K$ with $\varphi \simeq\left\langle a_{1}, \ldots, a_{m}\right\rangle$. Then $\Lambda^{k} \varphi$ is a symmetric bilinear form of dimension $\left(\begin{array}{c}m \\ k\end{array}\right)$ and

$$
\Lambda^{k} \varphi \simeq \underset{1 \leq i_{1}<\cdots<i_{k} \leq m}{ }\left\langle a_{i_{1}} \ldots a_{i_{k}}\right\rangle
$$

In particular, $\Lambda^{k}(m \times\langle 1\rangle) \simeq\left(\begin{array}{l}m \\ k\end{array}\right) \times\langle 1\rangle$.

Remark 5.4. We also have that $\Lambda^{k}(m \times\langle-1\rangle)=\left(\begin{array}{c}m \\ k\end{array}\right) \times\left\langle(-1)^{k}\right\rangle$.

Proposition 5.5. [7, Proposition 7.3] Let $\varphi$ and $\psi$ be symmetric bilinear forms over $K$ and let $k \in \mathbb{N}$. Then

$$
\Lambda^{k}(\varphi \perp \psi) \simeq \underset{i+j=k}{\perp} \Lambda^{i} \varphi \otimes \Lambda^{j} \psi
$$

5.1. Exterior powers of hyperbolic forms. We now compute exterior powers of a hyperbolic form $\phi \simeq h \times \mathbb{H}$ where $h \in \mathbb{N}$.

Proposition 5.6. Let $\phi \simeq h \times \mathbb{H}$ where $h \in \mathbb{N}$ and $k$ odd with $1 \leq k \leq 2 h-1$. Then

$$
\Lambda^{k} \phi \simeq \frac{1}{2}\left(\begin{array}{c}
2 h \\
k
\end{array}\right) \times \mathbb{H} \text {. }
$$

Proof.

$$
\begin{aligned}
\Lambda^{k} \phi & \simeq \Lambda^{k}(h \times\langle 1\rangle \perp h \times\langle-1\rangle) \\
& \simeq \frac{\perp}{i+j=k} \Lambda^{i}(h \times\langle 1\rangle) \otimes \Lambda^{j}(h \times\langle-1\rangle) \\
& \simeq \frac{\perp}{i+j=k}\left(\begin{array}{l}
h \\
i
\end{array}\right) \times\langle 1\rangle \otimes\left(\begin{array}{c}
h \\
j
\end{array}\right) \times\left\langle(-1)^{j}\right\rangle \\
& \simeq \sum_{i \text { odd }}\left(\begin{array}{c}
h \\
i
\end{array}\right)\left(\begin{array}{c}
h \\
k-i
\end{array}\right) \times\langle 1\rangle \perp \sum_{i \text { even }}\left(\begin{array}{c}
h \\
i
\end{array}\right)\left(\begin{array}{c}
h \\
k-i
\end{array}\right) \times\langle-1\rangle .
\end{aligned}
$$


Since

$$
\sum_{i \text { odd }}\left(\begin{array}{l}
h \\
i
\end{array}\right)\left(\begin{array}{c}
h \\
k-i
\end{array}\right)=\sum_{i \text { even }}\left(\begin{array}{l}
h \\
i
\end{array}\right)\left(\begin{array}{c}
h \\
k-i
\end{array}\right)
$$

for $k$ odd and since $\Lambda^{k} \phi$ has dimension $\left(\begin{array}{c}2 h \\ k\end{array}\right)$, the result follows.

Proposition 5.7. Let $\phi \simeq h \times \mathbb{H}$ where $h \in \mathbb{N}, k=2 \ell$ and $0 \leq \ell \leq h$. Then

$$
\Lambda^{k} \phi=\Lambda^{2 \ell} \phi \simeq\left(\begin{array}{l}
h \\
\ell
\end{array}\right) \times\left\langle(-1)^{\ell}\right\rangle \perp \frac{1}{2}\left(\left(\begin{array}{l}
2 h \\
2 \ell
\end{array}\right)-\left(\begin{array}{l}
h \\
\ell
\end{array}\right)\right) \times \mathbb{H}
$$

Proof. We use induction on $h$ and $\ell$. Let $P(h, \ell)$ be the statement in the proposition. $P(h, 1)$ is true for all $h$ since

$$
\begin{aligned}
\Lambda^{2} \phi & \simeq 2\left(\begin{array}{l}
h \\
2
\end{array}\right) \times\langle 1\rangle \perp\left(\begin{array}{l}
h \\
1
\end{array}\right)^{2} \times\langle-1\rangle \\
& \simeq\left(\begin{array}{l}
h \\
1
\end{array}\right) \times\langle-1\rangle \perp \frac{1}{2}\left(\left(\begin{array}{c}
2 h \\
2
\end{array}\right)-\left(\begin{array}{l}
h \\
1
\end{array}\right)\right) \times \mathbb{H} .
\end{aligned}
$$

Consider $P(1, \ell)$. Here $\phi \simeq \mathbb{H}$ and $\ell \in\{0,1\}$. Now

$$
\Lambda^{0} \mathbb{H}:=\langle 1\rangle \simeq\left(\begin{array}{l}
1 \\
0
\end{array}\right) \times\left\langle(-1)^{0}\right\rangle \perp \frac{1}{2}\left(\left(\begin{array}{l}
2 \\
0
\end{array}\right)-\left(\begin{array}{l}
1 \\
0
\end{array}\right)\right) \times \mathbb{H}
$$

and

$$
\Lambda^{2} \mathbb{H}=\langle-1\rangle \simeq\left(\begin{array}{l}
1 \\
1
\end{array}\right) \times\langle-1\rangle \perp \frac{1}{2}\left(\left(\begin{array}{l}
2 \\
2
\end{array}\right)-\left(\begin{array}{l}
1 \\
1
\end{array}\right)\right) \times \mathbb{H}
$$

So $P(1, \ell)$ is true.

Inductive step: Let $m, n$ be integers, $0<m<h$. Assume $P(m, n-1)$ is true for $0 \leq n-1 \leq m$ or, equivalently, $1 \leq n \leq m+1$. The case $n=m+1$ gives $\Lambda^{2(m+1)}:=\langle 0\rangle$. Also, assume that $P(m-1, n)$ is true for $0 \leq n \leq m-1$. We prove $P(m, n)$ to be true 
for $0 \leq n \leq m$ :

$$
\begin{aligned}
\Lambda^{2 n} \phi= & \Lambda^{2 n}(m \times \mathbb{H}) \\
= & \Lambda^{2 n}((m-1) \times \mathbb{H} \perp \mathbb{H}) \\
\simeq & \frac{\perp}{i+j=2 n} \Lambda^{i}((m-1) \times \mathbb{H}) \otimes \Lambda^{j} \mathbb{H} \\
\simeq & \Lambda^{2 n}((m-1) \times \mathbb{H}) \perp \Lambda^{2 n-1}((m-1) \times \mathbb{H}) \otimes \mathbb{H} \perp \Lambda^{2 n-2}((m-1) \times \mathbb{H}) \\
\simeq & \left(\begin{array}{c}
m-1 \\
n
\end{array}\right) \times\left\langle(-1)^{n}\right\rangle \perp \frac{1}{2}\left(\left(\begin{array}{c}
2(m-1) \\
2 n
\end{array}\right)-\left(\begin{array}{c}
m-1 \\
n
\end{array}\right)\right) \times \mathbb{H} \perp\left(\begin{array}{c}
2(m-1) \\
2 n-1
\end{array}\right) \times \mathbb{H} \\
& \perp\left(\begin{array}{l}
m-1 \\
n-1
\end{array}\right) \times\left\langle(-1)^{n}\right\rangle \perp \frac{1}{2}\left(\left(\begin{array}{c}
2(m-1) \\
2(n-1)
\end{array}\right)-\left(\begin{array}{c}
m-1 \\
n-1
\end{array}\right)\right) \times \mathbb{H} \\
\simeq & \left(\begin{array}{c}
m \\
n
\end{array}\right) \times\left\langle(-1)^{n}\right\rangle \perp \frac{1}{2}\left(\left(\begin{array}{c}
2 m \\
2 n
\end{array}\right)-\left(\begin{array}{c}
m \\
n
\end{array}\right)\right) \times \mathbb{H}
\end{aligned}
$$

by $P(m-1, n), P(m, n-1)$ and Proposition 5.6 .

Remark 5.8. The above proposition has been proved for ordered fields in [7, Proposition 11.8]. The proof uses the signature of $\phi$ with respect to an ordering.

Remark 5.9. In [7] it was shown that when $K$ is an ordered field and $\phi$ a hyperbolic form then $\Lambda^{k} \phi$ is hyperbolic if and only if $k$ is odd. Proposition 5.7 shows that this is not true for fields in general. For example, for a field $K$ containing $\sqrt{-1}$ and $\phi \simeq 4 \times \mathbb{H}$, we have $\Lambda^{2} \phi=\left(\begin{array}{l}4 \\ 1\end{array}\right) \times\langle-1\rangle \perp \frac{1}{2}\left(\left(\begin{array}{l}8 \\ 2\end{array}\right)-\left(\begin{array}{l}4 \\ 1\end{array}\right)\right) \times \mathbb{H}=14 \times \mathbb{H}$.

5.2. Some properties of binomial coefficients. We use some properties of binomial coefficients in the subsequent section. Some of them are well-known, others not. All of them can be derived from first principles or by using identities to be found in [9, Chapter 1], for example. We list the properties here: 


$$
\begin{aligned}
& \left(\begin{array}{l}
r \\
s
\end{array}\right)+\left(\begin{array}{c}
r \\
s-1
\end{array}\right)=\left(\begin{array}{c}
r+1 \\
s
\end{array}\right), \\
& \left(\begin{array}{l}
r \\
s
\end{array}\right)-\left(\begin{array}{c}
r \\
s-1
\end{array}\right)=\frac{r+1-2 s}{r+1}\left(\begin{array}{c}
r+1 \\
s
\end{array}\right), \\
& \left(\begin{array}{l}
r \\
s
\end{array}\right)+\left(\begin{array}{c}
r \\
s-2
\end{array}\right)=\left(\begin{array}{c}
r+2 \\
s
\end{array}\right)-2\left(\begin{array}{c}
r \\
s-1
\end{array}\right), \\
& \left(\begin{array}{l}
r \\
s
\end{array}\right)-\left(\begin{array}{c}
r \\
s-2
\end{array}\right)=\frac{r+2-2 s}{r+2}\left(\begin{array}{c}
r+2 \\
s
\end{array}\right), \\
& \left(\begin{array}{l}
r \\
s
\end{array}\right)=\frac{r}{s}\left(\begin{array}{l}
r-1 \\
s-1
\end{array}\right) .
\end{aligned}
$$

5.3. Computation of exterior powers of the trace form of a symbol algebra. Let $K$ and $S$ be as in Section 1. From Propositions 2.1, 3.1 and 3.2, we have that the trace form of $S$ is

$$
T_{S} \simeq \begin{cases}n \times\langle 1\rangle \perp \frac{n^{2}-n}{2} \times \mathbb{H} \simeq\left\langle(-1)^{\frac{n-1}{2}}\right\rangle \perp \frac{n^{2}-1}{2} \times \mathbb{H}, & \text { if } n \text { is odd } \\ \langle n\rangle\left\langle 1, a, b,(-1)^{\frac{n}{2}} a b\right\rangle \perp \frac{n^{2}-4}{2} \times \mathbb{H}, & \text { if } n \text { is even }\end{cases}
$$

For the remainder of this section, we shall use "Hyp" to denote an unspecified number of hyperbolic planes. Thus we may restate the computed trace form of $S$ as

$$
T_{S} \simeq \begin{cases}n \times\langle 1\rangle \perp \mathrm{Hyp} \simeq\left\langle(-1)^{\frac{n-1}{2}}\right\rangle \perp \mathrm{Hyp}, & \text { if } n \text { is odd } \\ \langle n\rangle\left\langle 1, a, b,(-1)^{\frac{n}{2}} a b\right\rangle \perp \mathrm{Hyp}, & \text { if } n \text { is even }\end{cases}
$$

Proposition 5.10. Let $n$ be odd and $k$ an integer such that $0 \leq k \leq n^{2}$. Then

$$
\Lambda^{k} T_{S} \simeq \begin{cases}\left(\frac{n^{2}-1}{2}\right) \times\left\langle(-1)^{\frac{k-1}{2}}\right\rangle \perp \text { Hyp, } & \text { if } k \text { is odd } ; \\
\left(\begin{array}{c}
\frac{n^{2}-1}{2} \\
\frac{k}{2}
\end{array}\right) \times\left\langle(-1)^{\frac{k}{2}}\right\rangle \perp \text { Hyp, } & \text { if } k \text { is even } .\end{cases}
$$


Proof. Let $k$ be odd. Then

$$
\begin{aligned}
\Lambda^{k} T_{S} & =\Lambda^{k}\left(\frac{n^{2}-1}{2} \times \mathbb{H} \perp\left\langle(-1)^{\frac{n-1}{2}}\right\rangle\right) \\
& \simeq \frac{\perp}{i+j=k} \Lambda^{i}\left(\frac{n^{2}-1}{2} \times \mathbb{H}\right) \otimes \Lambda^{j}\left\langle(-1)^{\frac{n-1}{2}}\right\rangle \\
& \simeq \Lambda^{k}\left(\frac{n^{2}-1}{2} \times \mathbb{H}\right) \perp \Lambda^{k-1}\left(\frac{n^{2}-1}{2} \times \mathbb{H}\right) \\
& \simeq\left(\begin{array}{c}
\frac{n^{2}-1}{2} \\
\frac{k-1}{2}
\end{array}\right) \times\left\langle(-1)^{\frac{k-1}{2}}\right\rangle \perp \text { Hyp. }
\end{aligned}
$$

A similar computation for even $k$ yields the result.

Proposition 5.11. Let $n$ be even. We write $T_{S} \simeq q_{S} \perp m \times \mathbb{H}$ where $q_{S} \simeq\langle n\rangle\left\langle 1, a, b,(-1)^{\frac{n}{2}} a b\right\rangle$ and $m=\frac{n^{2}-4}{2}$. Then, for $0 \leq k \leq n^{2}$,

$$
\Lambda^{k} T_{S} \simeq \begin{cases}\left(\begin{array}{l}
m+1 \\
\frac{k-1}{2}
\end{array}\right) \times\left\langle(-1)^{\frac{n(k-1)}{4}}\right\rangle q_{S} \perp \text { Hyp, } & \text { if } k \text { is odd; } \\
\left(\begin{array}{c}
\frac{n^{2}}{2} \\
\frac{k}{2}
\end{array}\right) \times\langle 1\rangle \perp \mathrm{Hyp}, & \text { if } k \text { is even and } n \equiv 0 \quad(\bmod 4) ; \\
\left(1-\frac{2 k}{n^{2}}\right)\left(\begin{array}{c}
\frac{n^{2}}{2} \\
\frac{k}{2}
\end{array}\right) \times\left\langle(-1)^{\frac{k}{2}}\right\rangle \perp \mathrm{Hyp}, & \text { if } k \text { is even, } k \leq \frac{n^{2}}{2} \text { and } n \equiv 2 \quad(\bmod 4) ; \\
\left(\frac{2 k}{n^{2}}-1\right)\left(\begin{array}{c}
\frac{n^{2}}{2} \\
\frac{k}{2}
\end{array}\right) \times\left\langle(-1)^{\frac{k+2}{2}}\right\rangle \perp \mathrm{Hyp}, & \text { if } k \text { is even, } k>\frac{n^{2}}{2} \text { and } n \equiv 2 \quad(\bmod 4) .\end{cases}
$$

Proof. Case (i). Let $k$ be odd. Then

$$
\begin{aligned}
\Lambda^{k} T_{S} & =\Lambda^{k}\left(m \times \mathbb{H} \perp q_{S}\right) \\
& \simeq \Lambda^{k-1}(m \times \mathbb{H}) \otimes q_{S} \perp \Lambda^{k-3}(m \times \mathbb{H}) \otimes \Lambda^{3} q_{S} \perp \text { Hyp } \\
& \simeq\left(\begin{array}{c}
m \\
\frac{k-1}{2}
\end{array}\right) \times\left\langle(-1)^{\frac{k-1}{2}}\right\rangle \otimes q_{S} \perp\left(\begin{array}{c}
m \\
\frac{k-3}{2}
\end{array}\right) \times\left\langle(-1)^{\frac{k-3}{2}}\right\rangle \otimes\left\langle(-1)^{\frac{n}{2}}\right\rangle q_{S} \perp \text { Hyp. }
\end{aligned}
$$

When $n \equiv 0(\bmod 4)$ we have $-1 \in K^{\times 2}$ and so

$$
\Lambda^{k} T_{S} \simeq\left(\left(\begin{array}{c}
m \\
\frac{k-1}{2}
\end{array}\right)+\left(\begin{array}{c}
m \\
\frac{k-3}{2}
\end{array}\right)\right) \times q_{S} \perp \text { Hyp }=\left(\begin{array}{c}
m+1 \\
\frac{k-1}{2}
\end{array}\right) \times q_{S} \perp \text { Hyp. }
$$

On the other hand, when $n \equiv 2(\bmod 4)$,

$$
\Lambda^{k} T_{S} \simeq\left(\begin{array}{c}
m+1 \\
\frac{k-1}{2}
\end{array}\right) \times\left\langle(-1)^{\frac{k-1}{2}}\right\rangle q_{S} \perp \text { Hyp. }
$$

Hence, for $n$ even and $k$ odd, we have

$$
\Lambda^{k} T_{S} \simeq\left(\begin{array}{c}
\frac{n^{2}-2}{2} \\
\frac{k-1}{2}
\end{array}\right) \times\left\langle(-1)^{\frac{n(k-1)}{4}}\right\rangle q_{S} \perp \text { Hyp. }
$$


Case (ii). Let $k$ be even. Then

$$
\begin{aligned}
\Lambda^{k} T_{S} & =\Lambda^{k}\left(m \times \mathbb{H} \perp q_{S}\right) \\
& \simeq \Lambda^{k}(m \times \mathbb{H}) \perp \Lambda^{k-2}(m \times \mathbb{H}) \otimes \Lambda^{2} q_{S} \perp \Lambda^{k-4}(m \times \mathbb{H}) \otimes \Lambda^{4} q_{S} \perp \text { Hyp } \\
& \simeq\left(\begin{array}{c}
m \\
\frac{k}{2}
\end{array}\right) \times\left\langle(-1)^{\frac{k}{2}}\right\rangle \perp\left(\begin{array}{c}
m \\
\frac{k-4}{2}
\end{array}\right) \times\left\langle(-1)^{\frac{n}{2}}\right\rangle\left\langle(-1)^{\frac{k}{2}}\right\rangle \perp \text { Hyp. }
\end{aligned}
$$

When $n \equiv 0(\bmod 4)$ we have $-1 \in K^{\times 2}$ and so

$$
\begin{aligned}
\Lambda^{k} T_{S} & \simeq\left(\left(\begin{array}{c}
m \\
\frac{k}{2}
\end{array}\right)+\left(\begin{array}{c}
m \\
\frac{k-4}{2}
\end{array}\right)\right) \times\langle 1\rangle \perp \text { Hyp } \\
& =\left(\begin{array}{c}
m+2 \\
\frac{k}{2}
\end{array}\right) \times\langle 1\rangle \perp \text { Hyp } \\
& =\left(\begin{array}{c}
\frac{n^{2}}{2} \\
\frac{k}{2}
\end{array}\right) \times\langle 1\rangle \perp \text { Hyp. }
\end{aligned}
$$

On the other hand, when $n \equiv 2(\bmod 4)$ we have that $\left\langle(-1)^{\frac{n}{2}}\right\rangle \simeq\langle-1\rangle$ and so

$$
\begin{aligned}
\Lambda^{k} T_{S} & \begin{cases}\left(\left(\begin{array}{c}
m \\
\frac{k}{2}
\end{array}\right)-\left(\begin{array}{c}
m \\
\frac{k-4}{2}
\end{array}\right)\right) \times\left\langle(-1)^{\frac{k}{2}}\right\rangle \perp \text { Hyp, } & \text { if } k \leq \frac{n^{2}}{2} ; \\
\left(\left(\begin{array}{c}
m \\
\frac{k-4}{2}
\end{array}\right)-\left(\begin{array}{c}
m \\
\frac{k}{2}
\end{array}\right)\right) \times\left\langle(-1)^{\frac{k+2}{2}}\right\rangle \perp \text { Hyp, } & \text { if } k>\frac{n^{2}}{2}\end{cases} \\
& = \begin{cases}\left(1-\frac{2 k}{n^{2}}\right)\left(\begin{array}{c}
\frac{n^{2}}{2} \\
\frac{k}{2}
\end{array}\right) \times\left\langle(-1)^{\frac{k}{2}}\right\rangle \perp \text { Hyp, } & \text { if } k \leq \frac{n^{2}}{2} ; \\
\left(\frac{2 k}{n^{2}}-1\right)\left(\begin{array}{c}
\frac{n^{2}}{2} \\
\frac{k}{2}
\end{array}\right) \times\left\langle(-1)^{\frac{k+2}{2}}\right\rangle \perp \text { Hyp, } & \text { if } k>\frac{n^{2}}{2} .\end{cases}
\end{aligned}
$$

Example 5.12. By Proposition 5.11 with $n=4$ and $k$ odd, we get $\Lambda^{k} T_{S} \simeq\left(\begin{array}{c}7 \\ \frac{k-1}{2}\end{array}\right) \times q_{S} \perp$ Hyp. Since $\left(\begin{array}{l}7 \\ l\end{array}\right)$ is odd for $0 \leq l \leq 7$, it follows that $\Lambda^{k} T_{S} \simeq q_{S} \perp$ Hyp for $k$ odd, $1 \leq k \leq 15$. For $n=4$ and $k$ even we get $\Lambda^{k} T_{S} \simeq\left(\begin{array}{l}8 \\ \frac{k}{2}\end{array}\right) \times\langle 1\rangle \perp$ Hyp. Since $\left(\begin{array}{l}8 \\ l\end{array}\right)$ is even for $1 \leq l \leq 7$ it follows that $\Lambda^{k} T_{S}$ is hyperbolic. These conclusions confirm [10, Corollaire 2].

Remark 5.13. In general, for $n \equiv 0(\bmod 4)$ and $k$ even, it is not true that $\Lambda^{k} T_{S}$ is hyperbolic. For example, with $n=12, \Lambda^{16} T_{S} \simeq\langle 1\rangle \perp$ Hyp.

Remark 5.14. From Proposition 5.11 it follows that $\Lambda^{k} T_{S}$ is hyperbolic for $n$ even and $k \in\left\{n, \frac{n^{2}}{2}\right\}$. For, when $n \equiv 0(\bmod 4)$ we have

$$
\begin{aligned}
\Lambda^{\frac{n^{2}}{2}} T_{S} & \simeq\left(\begin{array}{c}
\frac{n^{2}}{2} \\
\frac{n^{2}}{4}
\end{array}\right) \times\langle 1\rangle \perp \text { Hyp } \\
& =2\left(\begin{array}{c}
\frac{n^{2}-2}{2} \\
\frac{n^{2}-4}{4}
\end{array}\right) \times\langle 1\rangle \perp \text { Hyp }
\end{aligned}
$$


and when $n \equiv 2(\bmod 4)$ the result follows directly from the formula for $\Lambda^{k} T_{S}$.

Remark 5.15. It follows from Proposition 5.11 that $\Lambda^{n^{2}} T_{S}$ is anisotropic when $n$ is even.

Remark 5.16. As a consequence of Proposition 5.11, $\Lambda^{k} T_{S}$ is hyperbolic when $n \equiv 0$ $(\bmod 4), p$ is an odd prime divisor of $n$ and $k \in\{2,4,8,2 p, 4 p, 8 p\}$.

\section{AcKNOWLEDGEMENTS}

I would like to thank David Lewis and Thomas Unger for their guidance in the preparation of this paper and for their many suggested improvements. I am also grateful to Science Foundation Ireland who funded this research under the Research Frontiers Programme (project no. 07/RFP/MATF191).

\section{REFERENCES}

[1] Bourbaki, N., Éléments de Mathématique, Livre II, Algèbre, Hermann, Paris, 1959.

[2] Draxl, P. K., Skew Fields, Cambridge Univ. Press, Cambridge, 1983.

[3] Fein, B., Gordon, B., Smith, J.H., On the representation of -1 as a sum of two squares in an algebraic number field, J. Number Theory 3 (1971) 310-315.

[4] Knus, M.-A., Merkurjev, A., Rost, M., Tignol, J.-P., The Book of Involutions, Amer. Math. Soc. Colloq. Publ., Providence, RI, 1998.

[5] Lam, T. Y., Introduction to Quadratic Forms over Fields, Grad. Stud. Math., Providence, RI, 2004.

[6] Lewis, D. W., Trace forms of central simple algebras, Math. Z. 215 (1994) 367-375.

[7] McGarraghy, S., Exterior powers of symmetric bilinear forms, Algebra Colloq. 9:2 (2002) 197218.

[8] Narkiewicz, W., Number Theory, World Scientific, Singapore, 1983.

[9] Riordan, J., Combinatorial Identities, Robert E. Krieger Publishing Company, New York, 1979.

[10] Rost, M., Serre, J.-P., Tignol, J.-P., La forme trace d'un algèbre simple centrale de degré 4, C.R. Math. Acad. Sci. Paris, 342 (2006) 83-87.

[11] Scharlau, W., Quadratic and Hermitian Forms, Springer, Berlin, 1985.

School of Mathematical Sciences, University College Dublin, Belfield, Dublin 4, Ireland

E-mail address: ronan.flatley@ucd.ie 